\title{
Lift and Drag Forces for a Sphere on a Flat Wall in Non-Newtonian Shear Flow
}

\author{
Yaroslav Ignatenko ${ }^{1, *}$, Oleg Bocharov ${ }^{1}$, and Roland $\mathrm{May}^{2}$ \\ ${ }^{1}$ Scientist, Novosibirsk Technology Center of Baker Hughes Incorporated, 630090, Kutateladze 4a, \\ Novosibirsk, Russia \\ ${ }^{2}$ Mechanical Engineer, Celle Technology Center of Baker Hughes Incorporated, Baker-Hughes- \\ Strasse 1, Celle, Niedersachsen 29221, Germany
}

\begin{abstract}
Parametrical study of perfect steady rolling and steady state freely motion of spherical particle on the wall in shear flow of nonNewtonian fluid was carried out. In the case of perfect rolling the jumps of forces acting on a sphere in the moment of changing particle velocity from zero to positive were observed. Non-Newtonian properties of fluid lead to decreasing of drag force coefficient for low particle's velocity and to opposite effect for high velocity relative to Newtonian fluid. There is no such significant effect for a lift force coefficient. Steady state particle's velocity $\left(F_{d}\left(V_{p s s}\right)=0\right)$ increases for non-Newtonian fluid for both considered types of motion. Steady state particle's velocity and lift force coefficient are lower for the case of freely particle motion. Steady state rotational velocity decreases for non-Newtonian fluid.
\end{abstract}

\section{Introduction}

One of multiphase flow regimes is stirring or lifting of particles over a wall or a bed of particles. The movement of spherical particles in a shear flow along a wall is a common phenomenon in a nature and technical design. For example, the movement of river drift and the transport of cuttings during horizontal drilling can be described by this simple model. In these situations, the determination of forces acting on the particle is an important and fundamental task.

One of the first investigations about flow around a sphere near a wall was made by Goldman et al. 1967 [1, 2]. An analytical solution was obtained for the drag force and torque acting on the sphere near the wall using the Stokes approximation. Two scenarios were considered: a resting sphere in shear flow and a moving sphere in a non-moving fluid. In the latter scenario, the drag force and torque increases logarithmically with decreasing wall distance. Sphere motion on the wall was investigated numerically by Lee et al. [3, 4] using Navier-Stokes equations. They considered perfect rolling and free motion with creeping for a Newtonian fluid.

\footnotetext{
* Corresponding author: yaroslav.ignatenko@gmail.com
} 
Most articles presented in literature about rolling/sliding particles on a wall in shear flow are made for Newtonian rheology. Current study is concentrated on effect of non-Newtonian fluid rheology for the problem of single particle motion on a wall.

\section{Problem statements}

We consider a spherical particle with diameter $d$, rotating at an angular velocity $\omega$ about zaxis and translating parallel to a wall with velocity $v_{p}$ in $\mathrm{x}$-axis direction (see Fig. 1a). The far-field ambient flow is a linear shear flow directed in the x-axis $u_{\infty}=G y$, where $G$ is shear rate of undisturbed flow $\left(G=d u_{\infty} / d y\right)$. On the wall and sphere non-slip boundary conditions was set up. Steady state flow around the sphere can be described by Navier-Stokes equations

$$
\begin{gathered}
\nabla \cdot \mathbf{u}=0, \\
\mathbf{u} \cdot \nabla \mathbf{u}=-\frac{\nabla p}{\rho}+\nabla \cdot \boldsymbol{\tau},
\end{gathered}
$$

here $p$ - pressure, $\mathrm{u}$ - flow velocity, $\tau$ - viscous stress tensor, that depends on shear rate and is defined by rheological model. Here viscous stress tensor is described by the HerschelBulkley rheology law:

$$
\boldsymbol{\tau}=\left(k \dot{\gamma}^{n-1}+\tau_{0} / \dot{\gamma}\right) \dot{\gamma}
$$

where $\dot{\gamma}$ - shear rate tensor, $\dot{\gamma}=\sqrt{2 \dot{\gamma} \dot{\gamma}}$ - shear rate, $k$-consistency index, $n$-power index, $\tau_{0}-$ yield stress.

Simulation was carried out with Reynolds number $\operatorname{Re}_{s}=\frac{\rho G^{2} d^{2}}{2\left(k G^{n}+\tau_{0}\right)}$ of free stream up to 200 and positive particle's velocity $V_{p}=2 v_{p} d / G<1.4$ and made dimensionless by dividing the velocity of free stream on the opposite to particle center, power index $0.4 \leq n \leq 1.0$, Bingham number $B n=\tau_{0} / k G^{n}<10$. The dimensionless rotational velocity of the particle is $\Omega_{p}=\omega / G$.

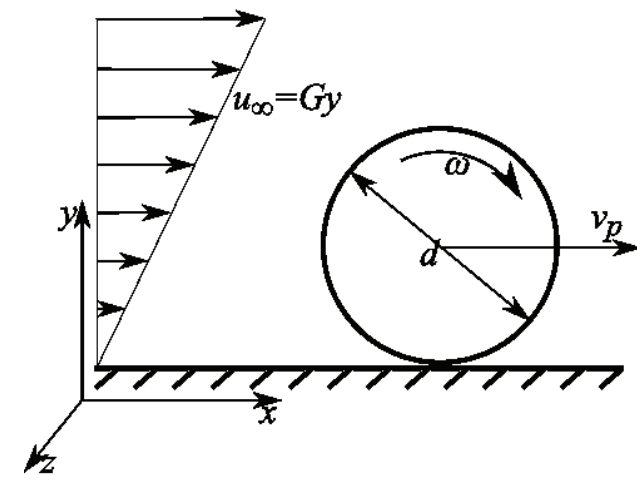

(a)

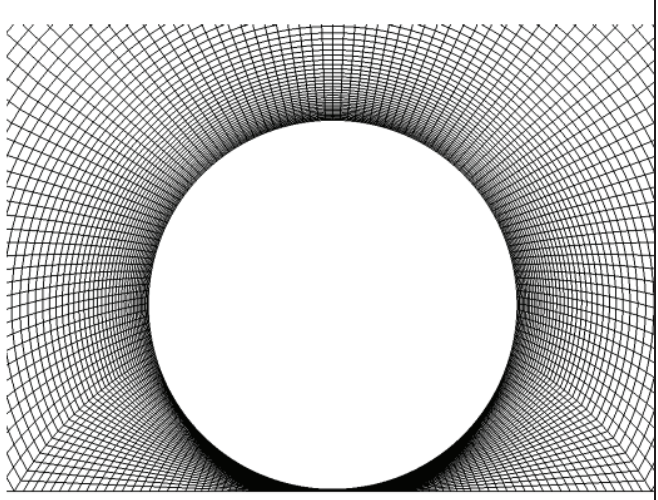

(b)

Fig.1. A schematic representation of a spherical rolling particle in a wall-bounded linear shear flow and the nomenclature used in this study (a), a sample of computational mesh around a sphere (b)

All simulations were made in the coordinate system translating with the particle using the open source CFD package OpenFOAM 3.0.1 [5] and the simpleFoam solver that is based on a SIMPLE-C algorithm [6]. The simulation volume was cuboid. Its surface was mapped isomorphically on the surface of a hemisphere, and then the surface of hemisphere was 
mapped isomorphically on surface of a particle (sphere). Using this technique a structural hexahedral mesh was created (Fig. 1b). The distance between a sphere and the boundaries of the simulation volume was equal to 25 sphere diameters. To avoid numerical issues, a small gap $d / 200$ between the sphere and the wall was presumed [2,3].

\section{Results and Discussion}

We considered two cases - perfect rolling and freely motion. Perfect rolling means that there is no creeping between particle and wall, angular velocity of sphere in this case is $\omega=2 \mathrm{Vp} / \mathrm{d}$. Freely motion indicates terminal motion of the particle when drag force and moment of forces acting on a sphere are equal to zero.

\subsection{Perfect rolling}

The simulation investigated the forces acting on the sphere and their dependence upon the flow regime and fluid rheology properties while a perfect rolling of a sphere on the wall. The results of our simulation are in a good agreement with results presented in [3, 4] for Newtonian fluids. When the particle translational velocity $V_{p}$ is low, the drag force coefficient $C_{d}$ in non-Newtonian fluid is lower than in Newtonian one (Fig. 2a). The opposite behavior of the drag force coefficient $C_{d}$ is observed while the translational velocity $V_{p}$ increases. This result can be explained by increasing of viscosity with decreasing of relative speed of the sphere. Lift force coefficient behavior was non-monotonic versus rheology parameters (Fig. 2b). Lift $C_{L}$ and drag $C_{d}$ forces increase in the vicinity of zero velocity $V_{p}$. This can be explained by significant changes in the flow regime: the transition from rest to a moving status of the particle.

In the article [3] the authors showed the existence of steady state velocity $V_{p s s}$ when the drag force $C_{d}$ is zero (free motion). The velocity $V_{p s s}$ increases with the Reynolds number. The results of our simulation show that in a non-Newtonian fluid the free motion velocity $V_{p s s}$ is more than in the Newtonian (Fig. 2a) and it can be greater than unity. In the case of large particle's velocity $V_{p}$, the perfect rolling lift force $C_{L}$ became negative (Fig. 2b). Consequently, friction between the particle and the surface prevent particle take-off in some cases.

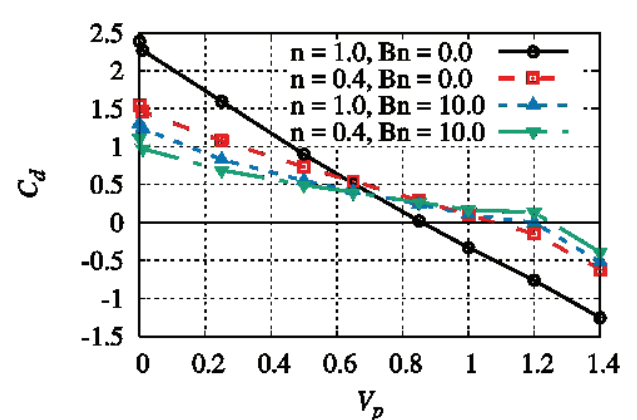

(a)

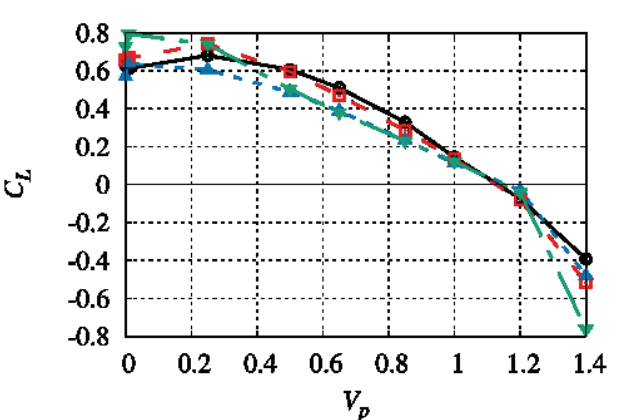

(b)

Fig.2. Forces dependence on translational velocity $V_{p}$ for $\operatorname{Re}_{s}=50$ and for different fluid properties (a) $C_{d}$; (b) $C_{L}$ 


\subsection{Freely motion}

We examined also the terminal steady-state of a particle when it is allowed for freely move in wall-bounded linear shear flow. This terminal state is characterized by zero-drag and zero-torque conditions. There is no friction between the sphere and wall because the gap is equal to $d / 200$. A simulation terminal angular velocity $\Omega_{p s s}$ and translational velocity $V_{p s s}$ were found (see Fig. 3). Terminal translational velocity $V_{p s s}$ increased with the increase of the Bingham number $B n$ and the decrease of the power index $n$ (Fig. 3a). In a friction-free case the terminal translational velocity $V_{p s s}$ can be greater than one, as in the case of perfect rolling.

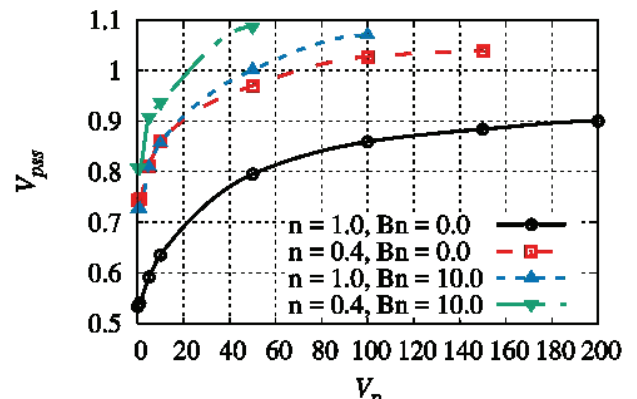

(a)

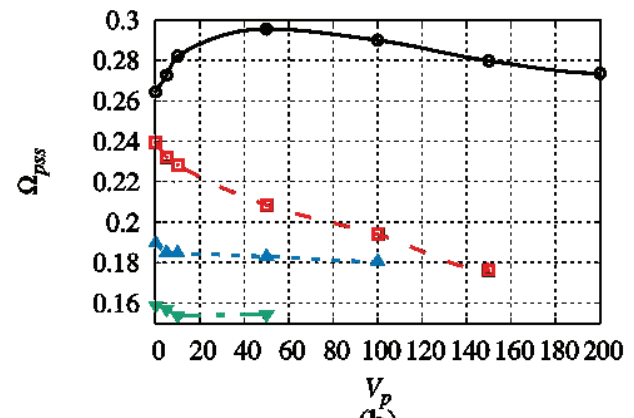

(b)

Fig. 3. Terminal translational velocity $V_{p s s}$ and rotational velocity $\Omega_{p s s}$ dependence on Reynolds number $\mathrm{Re}_{s}$ for different fluid properties

The terminal angular velocity $\Omega_{p s s}$ decreased with the decrease of the power index $n$ and with the increase of the Bingham number Bn (Fig. 3b). In addition, the increase of the Bingham number $B n$ flattened the angular velocity $\Omega_{p s s}$ dependency on Reynolds number $\mathrm{Re}_{s}$.

Dependence lift force $C_{L s s}$ on Reynolds number $R e_{s}$ and rheology parameters were obtained (Fig. 4). This provides the possibility to formulate take-off conditions for a spherical particle. Because the terminal velocity $V_{p s s}$ was greater in the non-Newtonian fluid, the lift force $C_{L s s}$ was lower (Fig. 3a, Fig. 4) because lift force decreases with translational velocity (Fig. 2b).

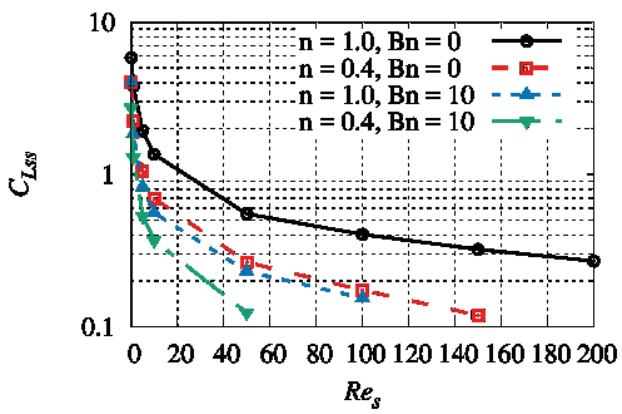

Fig. 4. Terminal lift force coefficient dependence $C_{L s s}$ on Reynolds number $\operatorname{Re}_{p}$ for different fluid properties

Non-Newtonian rheology of fluid leads to an increase of terminal velocity, to a decrease of terminal angular velocity and lift force.

The convergence of the numerical algorithm becomes worse with the increasing Reynolds $\operatorname{Re}_{s}$ and Bingham $B n$ numbers, the particle's velocity $V_{p}$ and the decreasing power index $n$. This is most likely due to the transient flow. 


\section{References}

1. A. J. Goldman, R. G. Cox, H. Brennen, Chem. Eng. Technol. 22, 637-651 (1967)

2. A. J. Goldman, R. G. Cox, H. Brennen, Chem. Eng. Technol. 22, 653-660 (1967)

3. H. Lee, S. Balachandar, J. Fluid Mech. 657, 89-125 (2010)

4. H. Lee, M. Y. Ha, S. Balachandar, Int. J. Multiphase Flow 37, 108-124 (2011)

5. Official cite of OpenFOAM URL: openfoam.org/

6. J. H. Ferziger, M. Peric, Computational Methods for Fluid Dynamics (Springer, Berlin, 2002) 